\title{
Accurate, high-coverage assignment of in vivo protein kinases to phosphosites from in vitro phosphoproteomic specificity data
}

\author{
Brandon M. Invergo ${ }^{1, *}$ \\ ${ }^{1}$ Translational Research Exchange @ Exeter, University of Exeter, Stocker \\ Road, Exeter EX4 4QJ \\ *Correspondence: b.invergo@exeter.ac.uk
}

\begin{abstract}
Phosphoproteomic experiments routinely observe thousands of phosphorylation sites. To understand the intracellular signaling processes that generated this data, one or more causal protein kinases must be assigned to each phosphosite. However, limited knowledge of kinase specificity typically restricts assignments to a small subset of a kinome. Utilizing simple machine-learning methods on data from high-throughput, in vitro kinase-substrate assays, I have developed an approach to high-coverage, multi-label kinase-substrate assignment called IV-KAPhE ("In vivo-Kinase Assignment for Phosphorylation Evidence"). Tested on human data, IV-KAPhE outperforms other methods of similar scope. Such computational methods generally predict a densely connected kinase-substrate network, with most sites targeted by multiple kinases, pointing either to unaccounted-for biochemical constraints or significant cross-talk and signaling redundancy. Finally, I show that such predictions can potentially identify biased kinase-site misannotations within families of closely related kinase isoforms.
\end{abstract}

\section{Introduction}

Protein phosphorylation is the most common form of post-translational modification and it plays a central role in intracellular signaling. Diverse protein kinases catalyze the binding of a phosphate group to an acceptor residue, typically serines $(\mathrm{S})$, threonines $(\mathrm{T})$ or tyrosines $(\mathrm{Y})$ in eukaryotes. The active sites of kinases' enzymatic domains exhibit phosphoacceptor-residue specificity, which can be broadly classified in eukaryotes as serine/threonine (S/T)-specific, tyrosine (Y)-specific, or so-called "dual-specificity" kinases. Kinase substrate-specificity is further determined by the protein primary and secondary structural contexts around the phosphoacceptor residue, as well as by allosteric structural mediation of docking (Ochoa et al., 2018; Bradley et al., 2021).

The sequence contexts around known phosphorylation sites ("phosphosites") have been widely used in computational approaches to predict new substrate sites of a protein kinase. Numer- 
ous methods have been developed to achieve this, employing, for example, scoring matrices (Yaffe et al., 2001; Obenauer et al., 2003; Miller et al., 2008; Jung et al., 2010; Safaei et al., 2011; Wagih et al., 2015; Krystkowiak et al., 2018), neural networks (Blom et al., 1999, 2004; Linding et al., 2007), support vector machines (Kim et al., 2004; Dou et al., 2014), sequence clustering (Zhou et al., 2004; Xue et al., 2011; Wang et al., 2020), kinase structure (Brinkworth et al., 2003), or grammatical inference (Datta and Mukhopadhyay, 2015). With the emergence of phosphoproteomics by liquid chromatography and tandem mass spectrometry (LC-MS/MS) enabling the routine detection thousands of phosphorylation sites in a single experiment (von Stechow et al., 2015), there is now little need to predict new phosphorylation sites in silico. The problem has instead changed to one of kinase-phosphosite assignment. Accordingly, new methods have emerged, based on models such as support vector machines (Zou et al., 2013; Yang et al., 2016), multiple kernel learning (Wang et al., 2017), Naïve Bayes (Ayati et al., 2019), networks (Wagih et al., 2016; Ma et al., 2020), and knowledge graphs (Nováček et al., 2020). Classical scoring matrices are often still used within these or related methods to model kinase substrate specificity (Wagih et al., 2016; Wang et al., 2017; Ma et al., 2020; Invergo et al., 2020).

Two major challenges face modern kinase-substrate assignment methods. First, dependence on literature-derived annotations for model training is subject to biases towards more commonly studied protein kinases (Invergo and Beltrao, 2018). This strongly limits and biases the kinases for which assignments can be made, whereas phosphoproteomic data requires unbiased, kinome-scale assignment. Second, many sites can be phosphorylated by more than one kinase (Hornbeck et al., 2015). However, a lack of complete, "all-versus-all" kinase-site training sets has prevented assignment methods from having been evaluated in such a multi-label setting. As a result, the predictive performance for promiscuous or strongly specific kinases can dominate evaluation metrics calculated across all kinase assignments.

Here, I describe improvements in multi-label, kinase-site assignment over previous methods covering a large subset of the human kinome. I achieve this without sophisticated machinelearning methods by focusing on the above considerations and building on the latest proteomescale, hypothesis-free data. The result is a nested model called IV-KAPhE which is built on models of in vitro kinase specificity and in vivo functional association. First I describe the approach used to construct the model and then I consider the hypotheses produced by it and other computational methods about the topology of the human phosphorylation network.

\section{Results}

I developed IV-KAPhE over three stages. First, I sought the best scoring matrix-based model to represent in vitro kinase sequence specificity. Next, I incorporated allosteric factors that mediate phosphorylation under the in vitro, context-free conditions of the training data. Finally, I nested the results of the in vitro model with features for predicting kinase-substrate functional associations in a predictor of in vivo phosphorylation (Figure 1). 


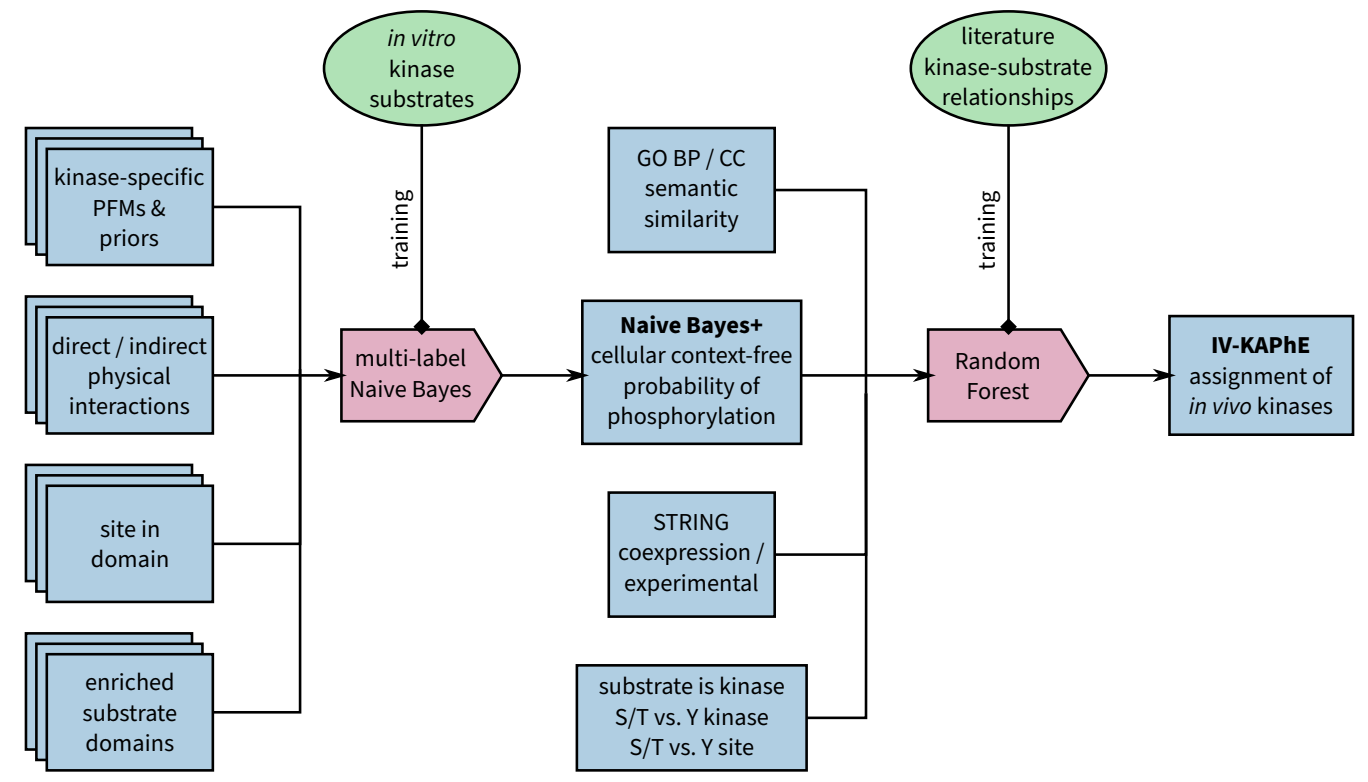

Figure 1: A schematic of the nested IV-KAPhE model for kinase-substrate assignment. Naïve Bayes+ consists of sub-models for each kinase, trained from kinase-specific, high-throughput in vitro kinase-substrate relationships. These sub-models together comprise a final, multi-label Naïve Bayes model. IV-KAPhE is a monolithic, dual-label Random Forest model trained from all literature-derived kinase-substrate annotations and random pairs as negative cases.

\section{Naïve Bayes is more appropriate than PSSM models for building a multi- label assignment method}

To construct specificity models for a large fraction of the human kinome, I used results from a recent phosphoproteomic, in vitro assay of kinase specificity (Sugiyama et al., 2019). In this experiment, protein extracts from HeLa cells were first treated with a thermo-sensitive protein phosphatase and then spiked with a recombinant protein kinase. The phosphorylated extracts were digested and subjected to phosphoproteomic analysis by LC-MS/MS. This provided in vitro substrates for 349 protein kinases, ranging from 1 to 1672 substrates per enzyme, with 322 kinases having at least 20 substrates. As a benefit of using a single cell line, each kinase was exposed to approximately the same set of potential substrates, detectable within the limits of random sampling by shotgun proteomics.

With this data, I sought to identify the best-performing specificity model on which to build a kinase-substrate assignment method. I first considered three scoring matrix-based specificity models: the position-frequency matrix (PFM) alone, the position-specific scoring matrix (PSSM) with log-likelihood ratios of the PFM to proteomic residue frequencies, and the log-likelihood ratio PSSM backed by position-specific phosphoproteomic residue frequencies. I hypothesized that the phosphoproteome-backed PSSM would be most appropriate for the task. Next, I took advantage of the of the fact that the design of the experiment provides truenegative kinase-site assignments to reformulate PFM-based specificity as a multi-label Naïve Bayes model (Zhang et al., 2009). I expected that the additional prior probability information would further strengthen assignments over PSSM models.

Breaking from convention (e.g. Wagih et al., 2015), for performing multi-label kinase-substrate 
assignment I retained the central residue in scoring. This allowed me to assign S/T and Y kinases simultaneously and to handle dual-specificity kinases cleanly. To strengthen the distinction between assignment to $\mathrm{S} / \mathrm{T}$ and $\mathrm{Y}$ kinases, I incorporated a position-weighting scheme into the models to provide greater scoring weight to highly resolved positions. I opted to use relative entropy for weighting as it provides better separation between well-defined and degenerate positions than information content does (Supplemental Figure S1). To simplify multi-label assignment, I aimed to use a single score cutoff for all kinases. Because each kinase's PFM is unique, the scores produced by PFMs or PSSMs will have different theoretical and empirical ranges for each kinase (Supplemental Figure S2). As a result, an effective score cutoff for one kinase might be outside the theoretical range for another kinase. To overcome this, I min-max normalized the PFM and PSSM scores to be between 0 and 1 (Wagih et al., 2015).

I evaluated the models via 10-fold cross-validation. I chose macro-averaged precision and recall (i.e. averaged across kinases) as evaluation metrics because the strong imbalance between positive and negative cases per kinase would de-emphasize false positives and inflate the area under the curve in Receiver Operator Characteristic (ROC) analysis (Davis and Goadrich, 2006). Using these metrics, I found that raw PFMs performed poorly, while PSSMbased methods and Naïve Bayes showed overall similar performance (Figure 2a). Nevertheless, phosphoproteome-backed PSSMs out-performed proteome-backed ones, confirming that a phosphoproteome background is preferable for kinase-substrate assignment. They also performed slightly better than Naïve Bayes, against my expectations.

The shapes of the precision-recall curves (Figure 2a) may appear strange compared to standard dual-label curves. They can be explained first by the strong imbalance between positive and negative cases per kinase, producing very low precision at low cutoffs (i.e. at high recall). Second, at more stringent cutoffs (low recall) a growing subset of kinases is no longer assigned to any sites, in which case precision is undefined and set to 0 for each kinase so affected. Thus, the "hump" in the macro-averaged precision-recall curves (Figure 2a) represents the point at which further gains in precision from more stringent cutoffs are offset by not only lower average recall but also a reduction in effective kinome coverage.

Turning to the macro-averaged F1 score (macro-F1), which assesses the balance between precision and recall, we see different relationships between macro-F1 and cutoff (Figure 2b). Notably, macro-F1 scores of scoring-matrix methods peak at specific cut-offs before dropping precipitously, whereas Naïve Bayes gives a consistent score across much of the cutoff range. This can be explained by observing the distributions of scores or probabilities produced by the different methods (Figure 2c). While the scoring-matrix methods produce many intermediate scores (particularly for $\mathrm{S} / \mathrm{T}$ kinases), Naïve Bayes mostly assigns probabilities close to 0 or 1 (Figure 2c). Moreover, the Naïve Bayes model better rejects inappropriate phosphoacceptors (e.g. Y phosphoacceptors for S/T kinases) in this way (Figure 2c). Thus, the PFM and PSSM-based models are particularly sensitive to the chosen score cutoff.

Naïve Bayes has the convenient property of a well-defined probability cutoff for assignment for all kinases $(P>0.5$; see Supplemental Methods). No such definition exists for the PSSM model. Given the PSSM models' sensitivity to cutoff selection, I sought to determine a robust selection method. As illustrated in Figure 2c, there is a sigmoidal relationship between phosphoproteome-backed PSSM scores and Naïve Bayes posterior probabilities, which could possibly be used to produce a PSSM score cutoff analogous to 0.5 posterior probability. Indeed, 

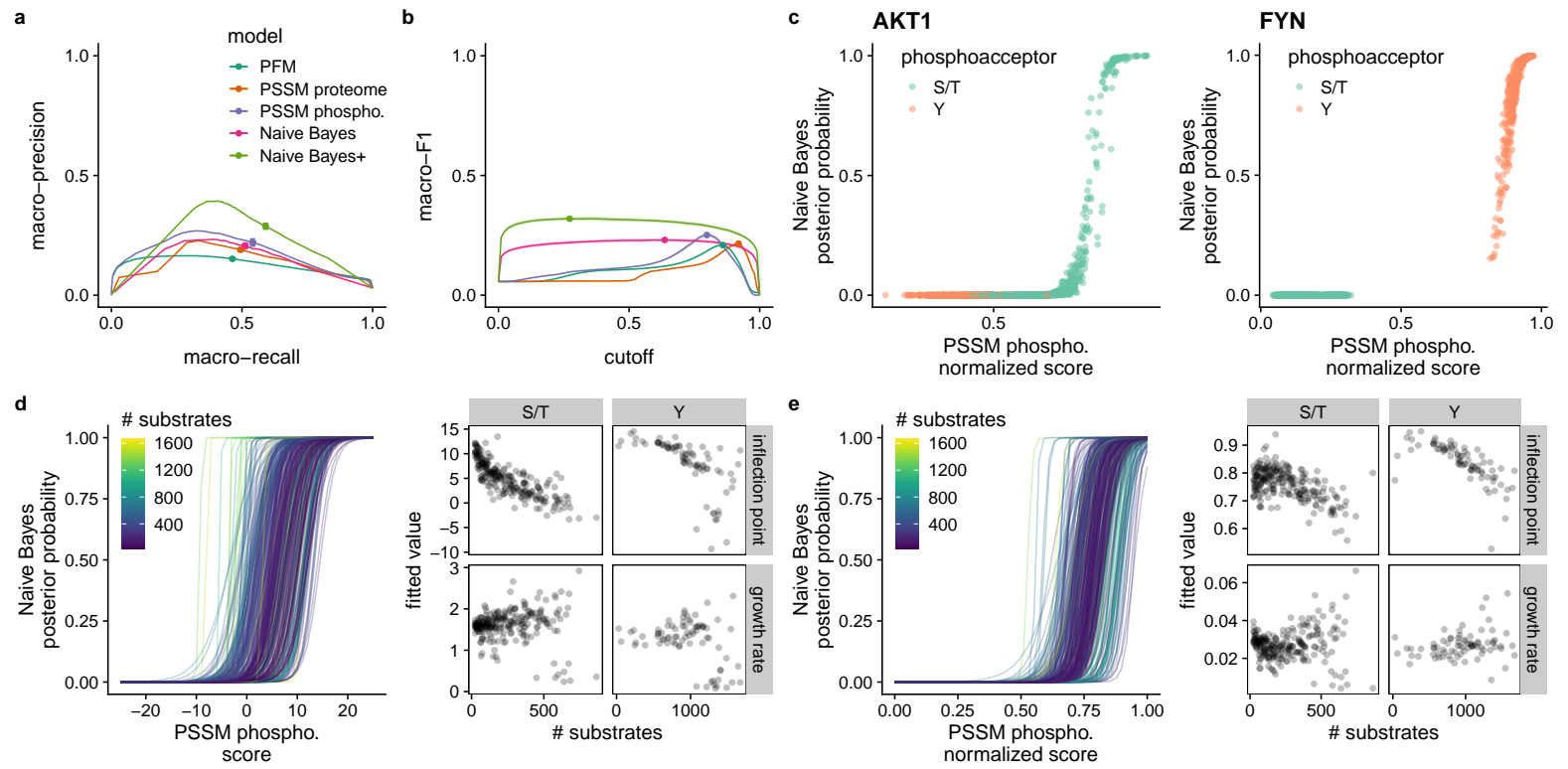

Figure 2: a) PSSM methods and Naïve Bayes perform similarly in cross-validation of multilabel kinase-substrate assignment via macro-averaged precision versus recall. The expanded Naïve Bayes+ model outperforms the other methods. Points indicate the scores at the cutoff that maximizes that macro-F1 score. Error bars showing 95\% confidence intervals at these points are indiscernible in most cases. b) The macro-averaged F1 scores behave differently with score/probability cutoff for scoring matrix-based models versus Naïve Bayes. PSSM and PFM-based models require a strictly defined cutoff. Naïve Bayes+ again outperforms the others and retains the same flat relationship with cutoff as basic Naïve Bayes. Points indicate the maximum value. Bands indicate the $95 \%$ confidence interval. Color assignments are the same as in (a). c) Example score distributions for a S/T kinase (AKT1) and a Y kinase (FYN) from one round of cross-validation. For S/T kinases, Naïve Bayes probabilities are largely distributed close to 0.0 and 1.0 while PSSM scores take more intermediate values, notably including scores for Y sites. Y kinases show better separation for both methods. d) Left: Logistic curves relating phosphoproteome-backed PSSM scores to Naïve Bayes probabilities. Each curve represents a fitted logistic function for each kinase. The color of the curve represents the number of kinase substrates used to fit each specificity model. Right: The fitted logistic curve parameters versus number of substrates. S/T and Y kinases have negative relationships between inflection point and numbers of substrates. e) Min-max normalization of PSSM scores does not produce a stable inflection point independent of the number of substrates. 
the phosphoproteome-backed PSSM score can be approximated by a logit transformation of the Naïve Bayes posterior probability (see Supplemental Materials). This approximation includes a dependency on the number of substrates used to fit a model, such that the 0.5-equivalent cutoff decreases with increasing number of substrates. In other words, because of varying training-set sizes, the log-likelihood PSSM score at which the foreground evidence effectively outweighs the background evidence also varies, whereas this is stabilized through normalization against total probability in Naïve Bayes.

To verify this, for each kinase I fit a logistic curve to their PSSM scores versus Naïve Bayes posterior probabilities calculated on a high-confidence set of human phosphosites (Ochoa et al., 2020) (Figure 2d). The fitted inflection point provides the PSSM score that is equivalent to a posterior probability of 0.5 . As predicted, I observed a strong dependence between this inflection point and the number of substrates used to fit the models, which differed with kinase type. I then checked whether min-max normalization of the PSSM scores remedies the problem (Figure 2e). For S/T kinases, the inflection points were generally around 0.75 , which is close to the observed macro-F1-maximizing cutoff of 0.803 (Figure 2b). However, both kinase types still had a decreasing inflection point with increasing number of substrates.

Together, these results suggest first that a raw PFM (as used in, e.g., Yang et al., 2016) is the weakest model. PSSMs perform best with a position-specific phosphoproteome background, but they require kinase-specific cutoffs, even after normalization, for multi-label assignment. The Naïve Bayes model offers good performance and a stable and universal cutoff. It is thus a better foundation for incorporating other features.

\section{Allosteric regulation features improve in vitro Naïve Bayes predictive per- formance}

Kinases can be optimized to bind with their substrates by allosteric mechanisms outside the enzymatic kinase domain, which may be exploited to improve the model. Given the Naïve Bayes assumption of feature independence, it is trivial to incorporate other features. Thus, I constructed a second Naïve Bayes model ("Naïve Bayes+") with added features based on: proteins physically interacting, directly or indirectly, with the kinase; proteins carrying a domain that is enriched among the kinase's substrates or interactors; and the phosphosite being within a protein domain. These features were modeled as Bernoulli-distributed, and each showed an overall difference across the kinome in empirical probability among substrates versus non-substrates (Figure 3).

In cross-validation, the Naïve Bayes+ model produces superior macro-averaged precision and recall than the other methods (Figure 2a). Its F1 score is similarly higher, while exhibiting the same flat behavior as the sequence-only Naïve Bayes model (Figure 2b). Given this improvement in performance I carried the Naïve Bayes+ model forward for nesting into the in vivo model. 


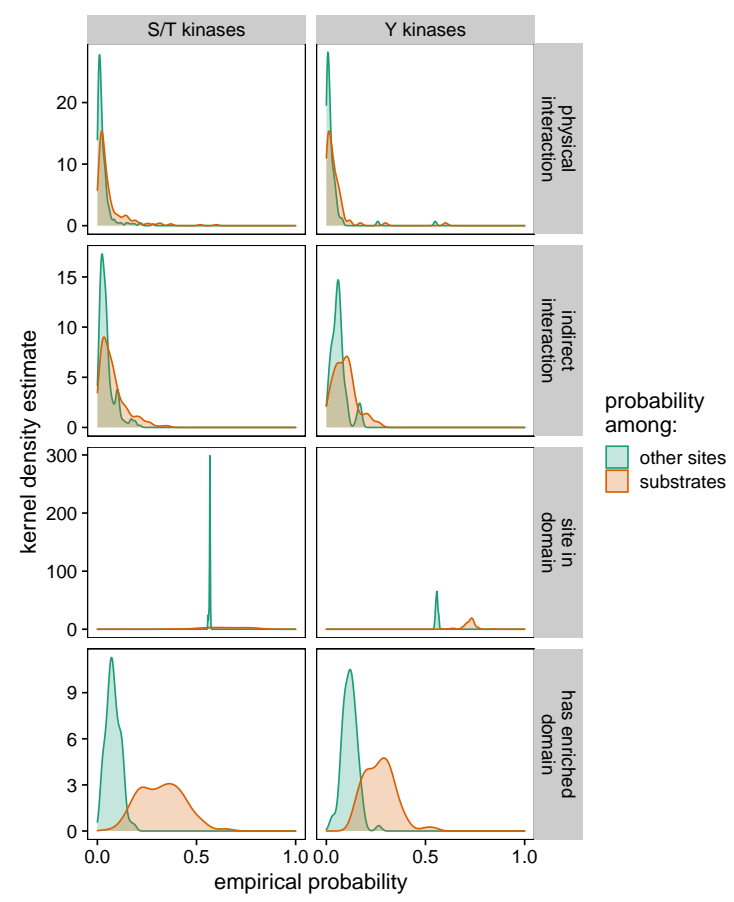

Figure 3: Distributions of empirical probabilities of four additional predictive features of in vitro kinase substrates. Distributions are represented by the kernel density estimate of the kinases' respective Bernoulli probability parameters. Each feature trends towards higher probability in in vitro substrates than in other sites.

\section{IV-KAPhE produces accurate multi-label assignment of in vivo kinases to phosphosites}

I constructed an in vivo kinase-substrate assignment method using the Random Forest model on five features for protein-protein functional association (Figure 1): the kinase-specific Naïve Bayes+ posterior probability for the site; the semantic similarities between the Gene Ontology (GO) "biological process" (BP) and "cellular component" (CC) annotations of kinase and substrate; and coexpression and high-throughput experimental scores between kinase and substrate from the STRING database (von Mering et al., 2005; Szklarczyk et al., 2019). Other features from STRING were discarded either by feature importance analysis (gene fusion, genome co-occurence, conserved neighborhood) or to avoid potential circularity with the training set (database imports, text-mining, combined score). I added three further features to strengthen possible discrepancies in performance (Figure 1): whether or not the substrate is itself a kinase, necessary because the functional association scores are symmetrical and can therefore produce false positives if the enzymatic roles are, in fact, reversed; whether the kinase is a S/T or Y kinase, to account for kinase-type differences in Naïve Bayes+ probability distributions; and the phosphoacceptor type (S/T or Y), for similar reasons.

For brevity, I will call this model "IV-KAPhE" ("In Vivo-Kinase Assignment for Phosphorylation Evidence"). For training IV-KAPhE, I took advantage of the fact that all kinase-specific substrate specificity information was used when training the underlying Naïve Bayes+ model. I thereby trained IV-KAPhE as a monolithic, non-kinase-specific model on all known kinase- 
substrate relationships annotated in the PhosphoSitePlus database (Hornbeck et al., 2015) and on an equally sized set of random assignments of kinases to high-confidence phosphorylation sites (Ochoa et al., 2020) as negative cases. During training, feature importance analysis revealed the Naiive Bayes+ posterior probability, the STRING "experimental" score, and GO BP semantic similarity to be the most important (Figure 4a). The features accounting for substrate kinases, $\mathrm{S} / \mathrm{T}$ versus $\mathrm{Y}$ kinases, and $\mathrm{S} / \mathrm{T}$ versus $\mathrm{Y}$ sites carried low importance but their omission worsened performance, particularly for $\mathrm{Y}$ kinases.

IV-KAPhE is not explicitly multi-label and the training set is likely incomplete (missing additional, true assignments between the kinases and sites), preventing full multi-label evaluation. I nevertheless evaluated it using multi-label metrics as described above. Overall, the model showed strong performance in cross-validation, reaching an average precision of 0.788 and recall of 0.745 at its nominal probability cutoff of 0.5 (Figure $4 b$ ). The macro-F1 score at this cutoff, 0.748 , was near-maximal (Figure 4b). Relaxing the cutoff slightly would improve recall, and thus F1, without significant loss of precision.

I next evaluated IV-KAPhE's performance on an external data set. To achieve this, I collected kinase-substrate relationships identified using the ProtMapper method from databases and textmining (Bachman et al., 2019), omitting any sites already present in the PhosphoSitePlus training set. I then matched these 5828 previously unseen relationships with an equal number of random kinase-site relationships.

I compared IV-KAPhE to the in vitro Naïve Bayes+ and phosphoproteome-backed PSSM models, as well as to other previously published methods with similar kinome coverage. To the best of my knowledge, the methods that meet that criterion are GPS 5.0 (Wang et al., 2020) and LinkPhinder (Nováček et al., 2020). I also compared it to NetworKIN 3.0 (Linding et al., 2007; Horn et al., 2014), as it shares a similar in vitro/in vivo hierarchical structure as IV-KAPhE. Assignments from GPS were as selected by the software's default, "medium"-stringency behavior, because each kinase requires a different score cutoff. For LinkPhinder and NetworKIN, I evaluated performance over a range of cutoffs, with a focus on LinkPhinder's published highstringency cutoff of 0.672 (Nováček et al., 2020) and NetworKIN's nominal likelihood-ratio cutoff of 1.0 (Horn et al., 2014). Each method covered a different, incomplete subset of the kinases in the test set (Figure 4c). NetworKIN has a significantly smaller coverage than the other methods. In order not to penalize models for coverage, each one was evaluated only on the subset of kinases that it could assign.

Like the training set, this test set is likely incomplete and cannot be fully evaluated in an allversus-all multi-label sense. Therefore, only those relationships explicitly annotated in the test set were evaluated. I first looked at per-kinase F1 score performance, which underlies the macro-averaged metric (Figure 4d). From this view, it is clear that IV-KAPhE produces largely consistent, high F1 performance across both S/T and Y kinases compared to the other methods. NetworKIN, GPS, and LinkPhinder all exhibit highly varied performance, with GPS notably showing weaker performance for Y kinases. PSSMs and Naïve Bayes+ likewise show varied performance and weak Y-kinase performance. Note that kinases with few test-case sites tend to cluster near 0 and 1 due to lack of resolution in calculating precision and recall.

In macro-averaged precision and recall, LinkPhinder performed only as well as the simpler, in vitro PSSM and Naïve Bayes+ models (Figure 4e). GPS exhibited stronger precision than 

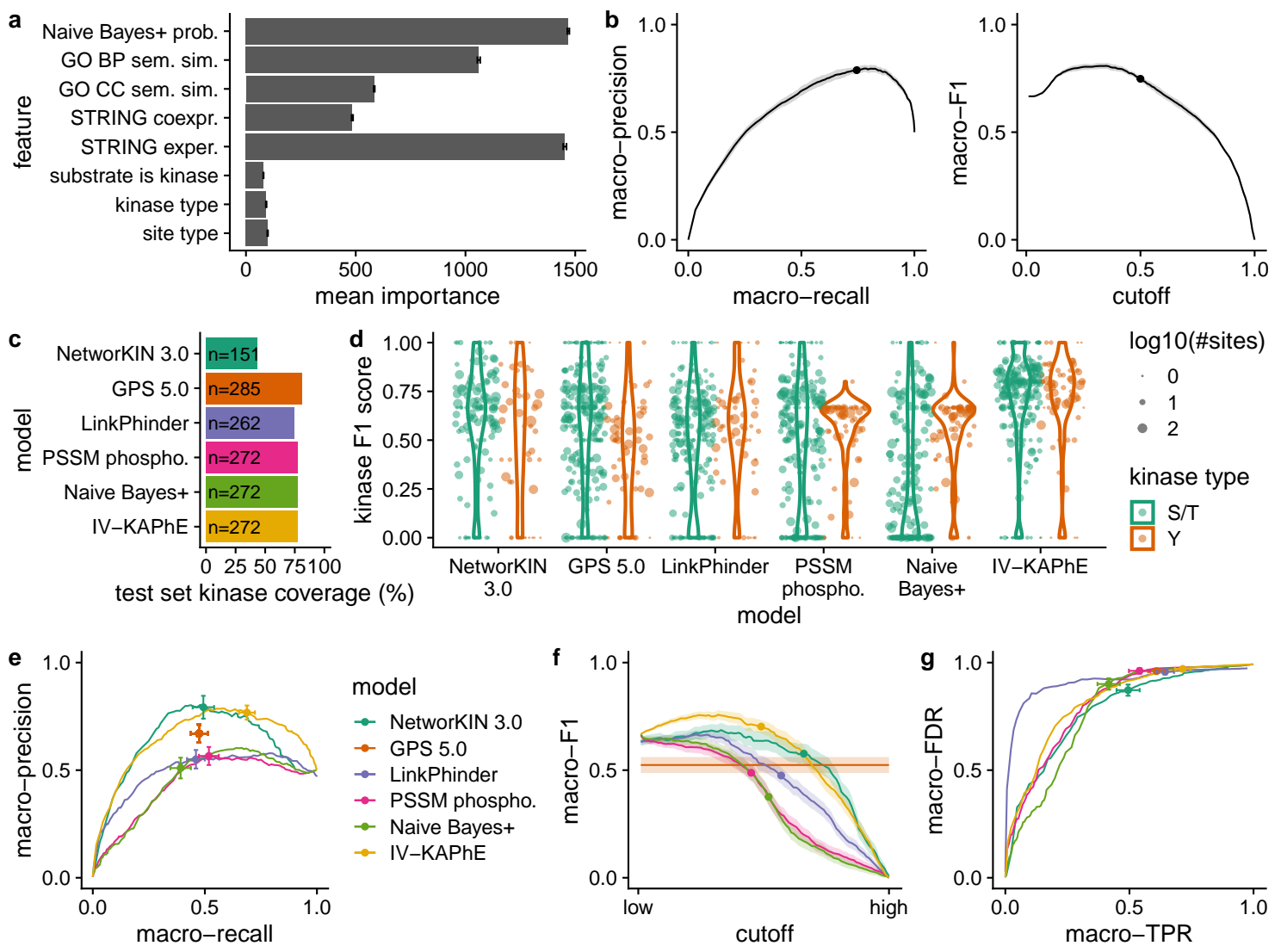

Figure 4: a) Naïve Bayes+ posterior probability, GO BP semantic similarity, and STRING experimental score had the greatest importance when training the Random Forest models. Error bars show standard error across cross-validation runs. b) Cross-validation evaluated via macroaveraged precision, recall and F1 all reflect strong performance by IV-KAPhE. c) IV-KAPhE's coverage of the external test data set is similar to GPS 5.0 and LinkPhinder. d) Kinase-specific F1 scores reveal IV-KAPhE's consistently strong performance across most kinases, with similar performance for S/T and Y kinases, compared to other methods. e) IV-KAPhE outperforms the simpler PSSM-based and Naïve Bayes+ methods as well as other previously published methods in kinase-substrate assignment of an external validation set. Points indicate the scores for simple assignments (GPS) or the scores at nominal cutoffs for quantitative predictions (cutoffs - IV-KAPhE: 0.5, PSSM: 0.75, Naïve Bayes+: 0.5, LinkPhinder: 0.672 (Nováček et al., 2020), NetworKIN 3.0: 1.0 (Horn et al., 2014)). Error bars show the 95\% confidence intervals at these points. f) IV-KAPhE has a higher macro-averaged F1 score than the other methods. Points and color assignments are as in (e). Bands indicate the 95\% confidence interval. g) Focusing on multi-label assignment for sites in the test set with known kinases, the macro-averaged false discovery rate (FDR; i.e. rate of novel assignments) dominates the average true positive rate (TPR). The curves are similar for most methods. At its nominal cutoff, IV-KAPhE has the highest FDR, but it is matched by the highest TPR. 
these models. NetworKIN and IV-KAPhE together showed the best precision, but IV-KAPhE provided it with superior recall and averaged across a much larger portion of the kinome (Figure 4e). Returning to the macro-F1 score, we similarly see that IV-KAPhE better balances precision and recall than the other methods (Figure 4f).

I next evaluated how many novel assignments are generated by the methods when performing all-versus-all kinase-site assignment. Considering only the kinases assignable from the test set and only the test-set sites for which at least one true kinase had been assigned, I compared the macro-averaged false discovery rate (FDR) and true positive rate (TPR) across the different methods. Here "false discovery rate" is a misnomer, as we do not know whether these assignments are true or false. For all models, FDR dominates the TPR: while the fraction of known cases correctly assigned may be high, the fraction of assignments that are novel is much higher (Figure 4g). IV-KAPhE's high FDR is nevertheless matched by a higher TPR than the other models. Thus, although IV-KAPhE also produces many novel assignments, we have a greater expectation of precision in its predictions

\section{Computational assignments hypothesize widespread signaling cross-talk and redundancy}

The topology of the human phosphorylation network is largely unresolved and biased towards commonly studied kinases (Hornbeck et al., 2015; Invergo and Beltrao, 2018). One open question is to what degree sites are phosphorylated by few kinases, as illustrated in canonical signaling pathway maps, versus multiple kinases through signaling noise, redundancy, or cross-talk between pathways. By applying a near kinome-scale kinase-site assignment model to a set of phosphosites representative of the phosphoproteome, we can produce hypotheses for such questions. Accordingly, I generated all-versus-all kinase-substrate assignments for a highconfidence human phosphoproteome (Ochoa et al., 2020) using Naïve Bayes+ and IV-KAPhE (Supplemental Table S1). I compared these to literature-derived assignments from PhosphoSitePlus and to LinkPhinder's assignments for all sites in PhosphoSitePlus (high-stringency cutoff).

Most sites in PhosphoSitePlus have a single kinase assigned to them and very few have more than four assigned kinases (Figure 5a). Thus, from the literature, we would suppose that multiple kinases rarely phosphorylate the same site. However this resource contains little data for understudied kinases such as isoforms of more commonly studied ones, which tend to have similar sequence specificities (Invergo and Beltrao, 2018; Bradley and Beltrao, 2019).

In contrast, Naïve Bayes+ predicts that most $\mathrm{S} / \mathrm{T}$ sites are phosphorylated by multiple kinases, but generally fewer than 10 (Figure 5a). However, it also predicts that a subset of S/T sites can be phosphorylated by around 20 different kinases. Conversely, the method assigns most tyrosine kinases to each Y site, pointing to a clear technical shortcoming of the in vitro model: cellular context plays the major role in determining Y kinase specificity. LinkPhinder and IV$\mathrm{KAPhE}$, on the other hand, both produce long-tailed distributions, with some sites having over 100 kinases assigned to them (Figure 5a). Reassuringly, the multi-modal distribution of Naïve Bayes+ is smoothed out by the biological context incorporated into IV-KAPhE, and it assigns fewer kinases to Y sites than Naïve Bayes+.

We can similarly ask whether specific proteins act as signaling hubs between pathways, with 

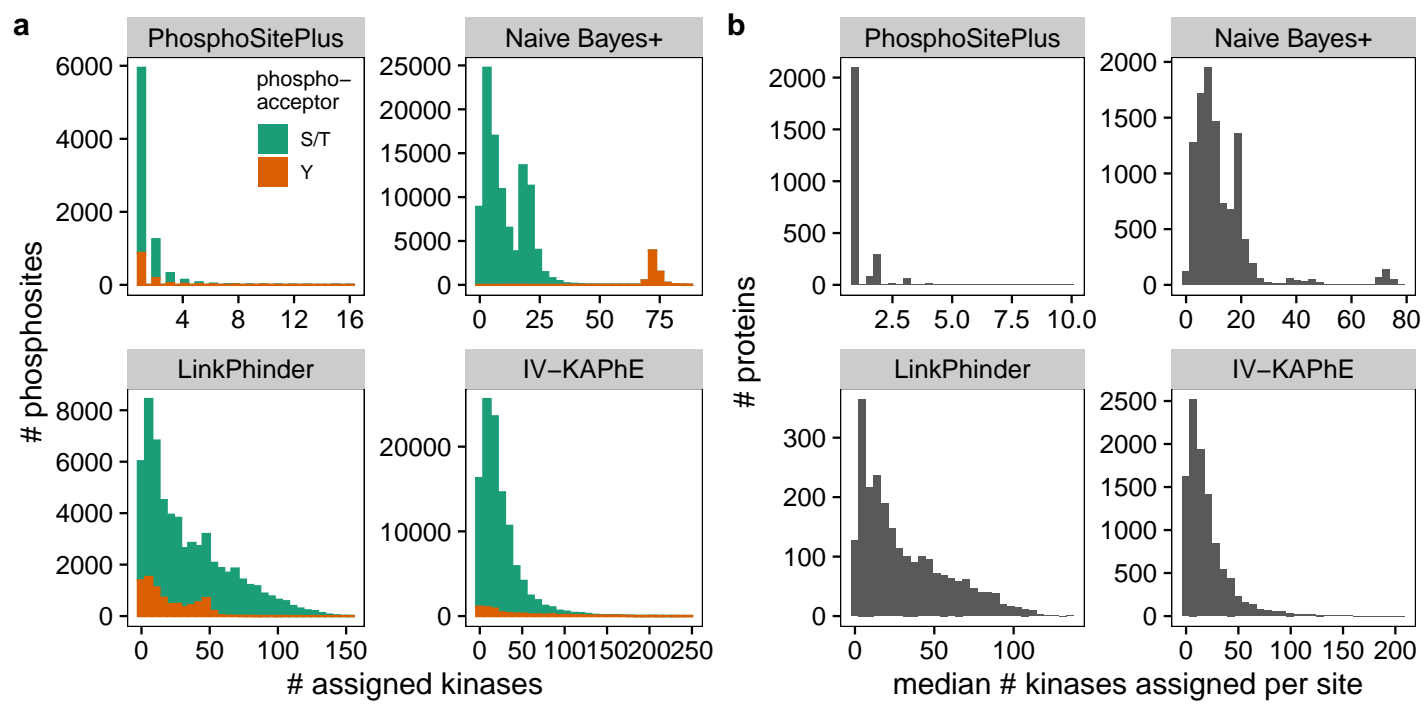

Figure 5: a) Histograms of the number of kinases associated with sites in the phosphoproteome reveal different views of the phosphorylation network. Literature annotations in PhosphoSitePlus suggest most sites are regulated by one or two kinases. In vitro Naïve Bayes+ predicts some S/T sites are "hubs" and all Y sites can be phosphorylated by most Y kinases. LinkPhinder and IV-KAPhE, in contrast, predict a long tail of hub sites. b) Histograms of the median number of kinases assigned per site for all proteins likewise show different predictions for hub proteins. Literature annotations suggest most proteins are phosphorylated by one kinase at each site. The computational methods all hypothesize multiple kinases per site, with some substrate proteins being very promiscuous at all their sites. 
multiple kinases phosphorylating each of their phosphosites. To answer this, I compared the median number of kinases assigned to sites for each substrate protein (Figure 5b). Again, literature-based assignments in PhosphoSitePlus suggest that most proteins are phosphorylated by a single kinase at each site. All three computational methods, on the other hand, propose the hypothesis that many proteins can be phosphorylated by multiple kinases at each of their sites (Figure 5b), i.e. that functional hubs on the protein-protein functional association network encounter many kinases, each with potentially similar and degenerate sequence specificity to the others.

One possible technical explanation of this for IV-KAPhE is that hub proteins' strong functional association scores may override low Naïve Bayes+ probabilities via some branches in the Random Forest model. This could arise from low, false-negative Naïve Bayes+ probabilities in the training set. As a result, IV-KAPhE would produce false positives for proteins occupying central positions in the network. For sites on such proteins, a post-hoc, stringent filter could be applied to select only assigned kinases with high Naïve Bayes+ scores.

\section{IV-KAPhE predictions identify possible misannotations of kinase isoform activity}

The composition of the human kinome is the result of extensive duplication events and is thus defined by families of kinases with highly similar sequence specificities (Bradley and Beltrao, 2019). Among closely related isoforms, often only one or two receive significant research attention, leading to an imbalance in kinase-substrate annotations among them (Invergo and Beltrao, 2018). Taking advantage of IV-KAPhE's broad kinome coverage, which is less biased in composition than literature annotations, I investigated patterns of substrate assignment among closely related isoforms.

First, I considered ribosomal protein S6 kinase alpha (S6K- $\alpha$ ) isoforms, among which isoforms 1 and 3 are the most commonly studied (Invergo and Beltrao, 2018). Using the full phosphoproteome assignments described in the previous section, I collected IV-KAPhE assignments of S6K- $\alpha$ isoforms for any site annotated in PhosphoSitePlus as being phosphorylated by at least one of them (Supplemental Table S2; Figure 6a). The bias towards annotations for S6K- $\alpha-1$ and -3 is plainly visible. There are a number of sites that IV-KAPhE predicts as being putative substrates of all six isoforms, pointing to multi-kinase phosphorylation, as well as some that are not well predicted for any isoform.

More interestingly, there are sites for which an isoform has stronger probability of phosphorylating the substrate than the annotated isoform(s). For example, site S103 on serum response factor (SRF; Uniprot: P11831) is annotated as a substrate of isoforms 3 and 5, whereas isoform 1 has the strongest evidence (Figure 6a). This annotation was derived from an in vitro study, in which the isoform used was not specified (Rivera et al., 1993). Two sites on Rho GTPaseactivating protein 31 (ARHGAP31; Uniprot: Q2M1Z3), S1106 and S1178, are annotated as substrates of isoform 1, whereas IV-KAPhE gives a stronger probability to isoform 2 (Figure $6 a)$. In this case, a S6K- $\alpha-1$ gene construct was used to induce phosphorylation under controlled conditions, while siRNAs targeting isoforms 1 and 3 were used to validate endogenous phosphorylation (Ben Djoudi Ouadda et al., 2018). Furthermore, site S1178 was not, in fact, tested, but rather merely identified as a putative site by $\mathrm{S} 6 \mathrm{~K}-\alpha$ sequence-motif analysis (Ben 


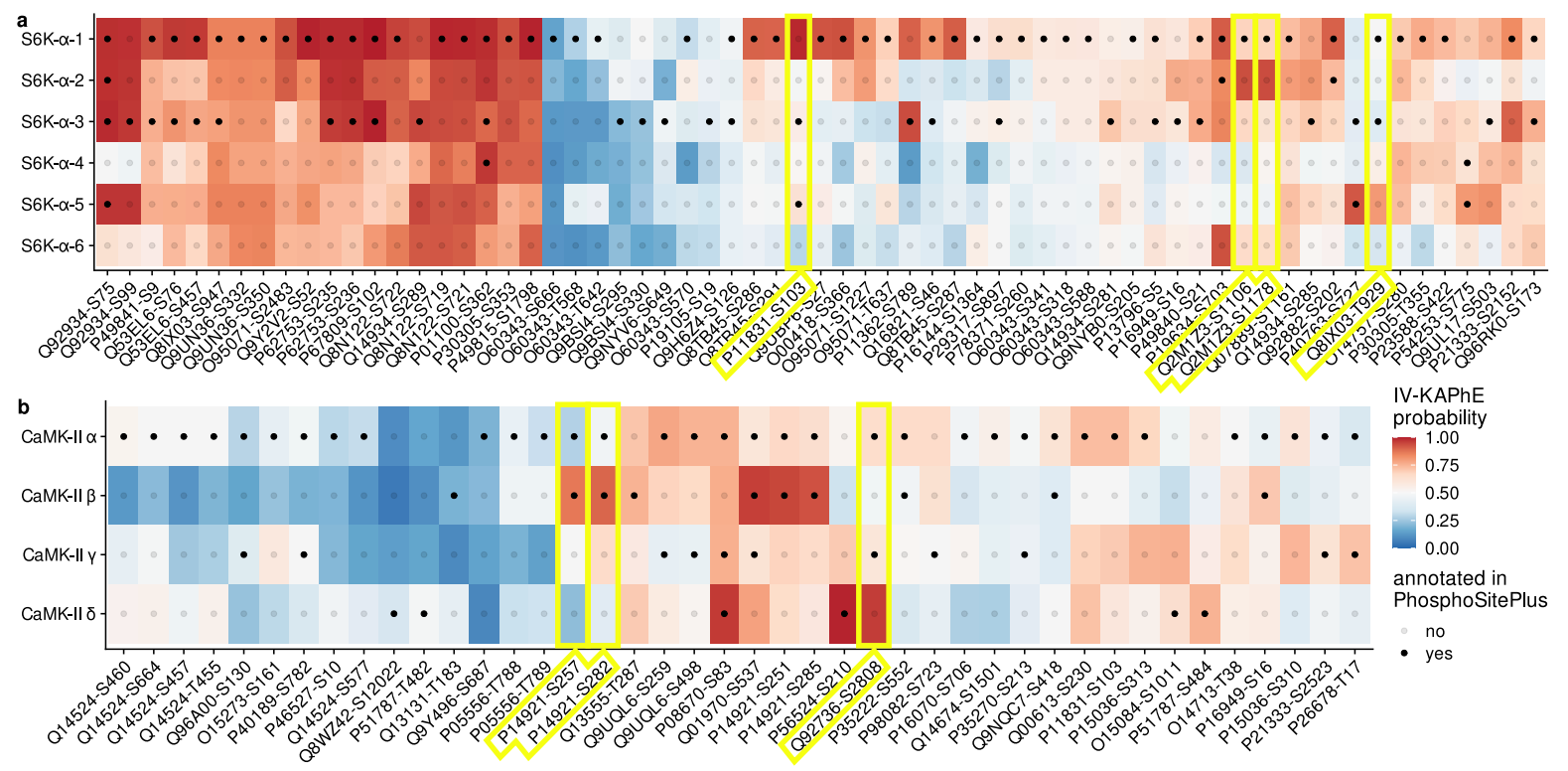

Figure 6: a) IV-KAPhE assignments of ribosomal protein S6 kinase alpha isoforms for all sites annotated in PhosphoSitePlus as being phosphorylated by at least one of the isoforms. Red colors indicate assignments predicted as likely by IV-KAPhE. Highlighted sites, discussed in the text, are examples that IV-KAPhE predicts are more likely to be phosphorylated by a different isoform than the one annotated. b) IV-KAPhE assignments of calcium/calmodulindependent protein kinase type II subunits to annotated sites, as described in (a).

Djoudi Ouadda et al., 2018). Finally, site T929 on protein KIBRA (WWC1; Uniprot: Q8IX03) is annotated to isoforms 1 and 3, however IV-KAPhE assigns low probabilities to both of these, instead favoring isoform 5 (Figure 6a). Here, the annotations are based on an in vitro analysis using recombinant isoforms 1 and 3 (Yang et al., 2014). By incorporating in vivo information, IV-KAPhE proposes the more likely causal isoform. I note that in many of these cases, IV-KAPhE assigns multiple kinases to the sites, albeit at varying degrees of probability, so the original assignments may be correct but incomplete.

I then carried out a similar analysis for calcium/calmodulin-dependent protein kinase type II (CaMK-II) subunits, which can form homo- or heteromultimeric holoenzymes, potentially complicating kinase assignment. As with $\mathrm{S} 6 \mathrm{~K}-\alpha$, some sites are assigned with similar probabilities to multiple subunits, while others point to possible misannotations (Supplemental Table S3; Figure 6b). For example, sites S257 and S282 on transcription factor C-ets-1 (ETS1; Uniprot: P14921) are both annotated to the most commonly studied subunit, $\alpha$, and the least commonly studied subunit, $\beta$ (Invergo and Beltrao, 2018), whereas IV-KAPhE does not assign the sites to the $\alpha$ subunit. In the associated study, phosphorylation was tested in vitro using the $\alpha$ subunit, while it was tested in vivo through expression of a $\beta-\gamma$ construct (Liu and Grundström, 2002). In a final example, site S2808 on ryanodine receptor 2 (RYR2; Uniprot: Q92736) is annotated to subunits $\alpha$ and $\gamma$, whereas IV-KAPhE most strongly assigns it to the $\delta$ subunit (Figure 6b). In this case, the subunit or subunits used in the original, in vitro experiment are not specified (Rodriguez et al., 2003). 


\section{Discussion}

In this work, I took advantage of the latest hypothesis-free, phosphoproteomic data in an effort to produce accurate, kinome-scale kinase-substrate assignments. Kinase-substrate annotations are not available for most species to the same level as humans or model species. Thus, kinase-substrate assignment methods that depend on such annotations cannot be applied to those species. The IV-KAPhE method presented here requires a high-throughput, phosphoproteomic kinase-substrate assay; high-throughput physical interaction data; Gene Ontology annotations, which are often have good coverage by orthology; and STRING scores, which are available for many species. This makes it a suitable method for kinase-site assignment in nonmodel species. While the first two requirements are, indeed, non-trivial and expensive, they are less onerous and time-consuming than low-throughput assays of individual kinase-substrates at a kinomic scale. Furthermore, the generality of the training data for the in vivo part of IV$\mathrm{KAPhE}$ means that it may be possible to use kinase-substrate annotations from other species as functional-association training data, a possibility that remains to be explored.

Even stringently assessed, the human phosphoproteome consists of over 100,000 different sites on at least 12,000 different proteins (Ochoa et al., 2020), of which only a fraction have literature-derived kinase assignments. These assignments are furthermore biased towards wellstudied protein kinases (Invergo and Beltrao, 2018). Thus the functional roles of many human protein kinases, including closely related isoforms of more commonly studied kinases, remain unknown. Applying IV-KAPhE predictions revealed the perils of these biases. On one hand, researchers use commonly studied isoforms for in vitro or artificial in vivo analysis, whereas the endogenous causal kinase may be a different isoform. On the other hand, when isoforms are not adequately specified in literature, annotators may default to inappropriately assigning the most commonly studied isoform to a site. As a result, the network mapping kinases to each human phosphosite remains not only largely unresolved, but its topology cannot be accurately extrapolated from existing literature-derived annotations.

Due to poor sequence conservation, many phosphosites are expected to be "off-target" and without function (Landry et al., 2009; Levy et al., 2012; Ochoa et al., 2020). Nevertheless, the methods presented here confidently assign multiple kinases to most sites. They collectively posit, based on the best available data, that this off-target noise is not due to low-probability events on non-optimized substrate sequences, as suggested by signaling dynamics (Kanshin et al., 2015). They thereby propose that the kinase-substrate network is densely connected. Incorporating further biochemical constraints into future models may reduce this apparent density and reject the computational hypothesis. Otherwise, the results will equally suggest that ontarget, functional phosphorylation also can generally be catalyzed by multiple kinases, raising the question of how kinase functional specialization is maintained across the human kinome.

\section{Methods}

\section{In vitro protein kinase specificity models}

For a detailed, mathematical descriptions of specificity model construction, see Supplemental Methods. They are described here in brief. All models were built from 15-residue sequence windows around the phosphoacceptor (+/-7 residues). Substrate sequences were weighted be- 
fore counting (Henikoff and Henikoff, 1994). Position-specific pseudocounts were added using the method of Henikoff and Henikoff (1996) and supplemented according to missing tail residues if sites localized to the 5' or 3' tail of the substrate protein. Column weights were calculated as the relative entropy versus background frequencies (PFM and phosphoproteomebacked PSSMs: position-specific residue frequencies from the full set of observed sequence windows; proteome-backed PSSMs: proteomic residue frequencies). PFM and PSSM scores were min-max normalized by calculating the theoretical minimum and maximum scores produced by the PSSM (Wagih et al., 2015).

The multi-label Naïve Bayes model was built using four components: the PFM constructed from a kinase's substrates; the PFM of all the substrates not phosphorylated by the kinase; and the prior probabilities of a site being phosphorylated by the kinase or by any other kinase. The priors were empirically estimated as the fraction of substrates in the experiment that were phosphorylated by the kinase or not, respectively. For determining the posterior probability that a kinase $K$ phosphorylates a site with sequence window $\mathbf{S}$, given PFM scores $s_{K}$ for $K$ and $s_{\bar{K}}$ for all other kinases and prior probabilities $P(K)$ and $P(\bar{K})$, the following was calculated:

$$
P(K \mid \mathbf{S})=\frac{s_{K} P(K)}{s_{K} P(K)+s_{\bar{K}} P(\bar{K})}
$$

A kinase was assigned to a site if $P(K \mid \mathbf{S})>0.5$.

The Naïve Bayes+ model further incorporated Bernoulli-distributed features with the PFMbased likelihood function. Probability parameters of substrates being direct or indirect interaction partners, of carrying domains enriched among a kinase's substrates or interaction partners, or of substrate sites being within a predicted protein domain were estimated empirically from the training data. Domain enrichment was calculated among substrates or interaction partners via Fisher's hypergeometric test and $p$-values were adjusted for false-discovery rate before being tested at a critical value of 0.05 .

\section{Multi-label cross-validation}

For multi-label performance evaluation, I performed 10 iterations of 10-fold cross-validation, restricting to kinases with models trained on at least 20 substrates. Both kinase substratedomain enrichment and sequence specificity models were recalculated from each fold's training subset. Performance was evaluated using macro-averaged (averaged over the set of all kinases, $\mathbb{K})$ precision, recall and $\mathrm{F} 1$ scores:

$$
\begin{aligned}
\text { prec } & =\frac{\sum_{K} \frac{T P_{K}}{T P_{K}+F P_{K}}}{|\mathbb{K}|} \\
\mathrm{rec} & =\frac{\sum_{K} \frac{T P_{K}}{T P_{K}+F N_{K}}}{|\mathbb{K}|} \\
\mathrm{F} 1 & =\frac{\sum_{K} \frac{2 \times \mathrm{prec} \times \mathrm{rec}}{\mathrm{prec}+\mathrm{rec}}}{|\mathbb{K}|}
\end{aligned}
$$


where $T P_{K}, F P_{K}$, and $F N_{K}$ are the true-positive, false-positive, and false-negative counts for kinase $K$, respectively. If a score was undefined due to division by 0 , it was set to 0 .

\section{Implementation}

Kinase specificity model training and scoring were implemented in and performed using a bespoke, free and open-source toolkit called motif-kit (https://www . gitlab.com/brandoninvergo/ motif-kit). The code was written in ANSI C99 for POSIX systems and is dependent only on the GNU Scientific Library (https://www.gnu.org/software/gsl) and the HDF5 library (https://www.hdfgroup.org/solutions/hdf5/), with unit tests further depending on the Check testing framework (https://libcheck. github.io/check/). All other methods (domain enrichment, multi-label CV, etc.) were implemented using R (version 4.1.0).

\section{IV-KAPhE Model}

\section{Model construction}

The IV-KAPhE model was built via the Random Forest method, as implemented in the R package "ranger" (version 0.13.1; Wright and Ziegler, 2017). The models were built with 500 trees. The variable importance mode (parameter "importance") was set to "impurity" (the Gini index) and the splitting rule (parameter "splitrule") was set to "gini". The model was trained to classify a given kinase-phosphosite pair as "true" (the kinase phosphorylates the site) or "false" (it does not) and set to provide a probability for each class. Feature selection was performed via variable-importance analysis, as implemented in ranger. The final list of features retained for model construction were: Naïve Bayes+ posterior probability, GO BP and CC semantic similarity, STRING coexpression and experimental scores, whether the substrate protein is itself a kinase, the kinase type (S/T or Y), and the site class (S/T or Y).

\section{Cross-validation}

Kinase-substrate relationships identified in the PhosphoSitePlus database (Hornbeck et al., 2015) were used as true positives in the training set for cross-validation. The same kinases from the true positive set (including multiple occurrences) were randomly assigned to other sites from the human phosphoproteome (Ochoa et al., 2020) to form a negative set. To this end, S/T kinases were randomly assigned to $\mathrm{S} / \mathrm{T}$ sites and $\mathrm{Y}$ kinases were assigned to $\mathrm{Y}$ sites. Additionally, any S/T or Y kinases that were annotated as phosphorylating the opposite site type in the true positive set were randomly assigned to a similar proportion of such sites in the negative set. In all cases, the proportion of substrate kinases observed in the positive set was maintained in the random negative set. Sites were filtered not to include sites found in the true positive training set or the external testing set (see below). 10-fold cross validation was performed and evaluated, restricted to kinases with Naive Bayes+ models trained on at least 20 substrates, via multi-label precision, recall, and F1 as described above. Cross-validation was performed ensuring that any kinase present in a fold had at least one positive and one negative site. 


\section{External Validation}

IV-KAPhE was trained using the full PhosphoSitePlus and random kinase-site pair training set as described above. An external evaluation set was prepared by identifying kinase-substrate relationships inferred via the ProtMapper method (Bachman et al., 2019) which were not present in the PhosphoSitePlus data set. These sites were accompanied by further random negative kinase-site pairs as described above. Predictions made by the model on this testing set were evaluated via multi-label precision, recall, and F1.

I furthermore evaluated the assignments for these kinase-site pairs made by phosphoproteomebacked PSSMs, Naïve Bayes+, and three other, previously published tools with similar kinomic scope or model architecture: NetworKIN 3.0 (Horn et al., 2014), GPS 5.0 (Wang et al., 2020), and LinkPhinder (Nováček et al., 2020). NetworKIN and GPS were run in-house with their default settings, whereas the LinkPhinder scores produced by the authors were used. For further evaluation, the published stringent LinkPhinder cutoff of 0.672 (Nováček et al., 2020) and the nominal NetworKIN cutoff of 1.0 (Horn et al., 2014) were used.

The test set was then re-evaluated with all methods, now allowing all-versus-all kinase-site assignments, restricted to sites that had at least one true kinase assigned. The rate of novel assignments was estimated via the macro-averaged false discovery rate (FDR) and compared to the macro-averaged true positive rate (identical to recall):

$$
\begin{aligned}
\mathrm{FDR} & =\frac{\sum_{K} \frac{F P_{K}}{T P_{K}+F P_{K}}}{|\mathbb{K}|} \\
\mathrm{TPR} & =\frac{\sum_{K} \frac{T P_{K}}{T P_{K}+F N_{K}}}{|\mathbb{K}|}
\end{aligned}
$$

"False positive" (FP) is used here by convention although it is a misnomer in the present case, as we do not know that the assignments are false.

\section{Kinase Assignment Distributions}

IV-KAPhE was retrained on a full training set consisting of the union of the PhosphoSitePlus and ProtMapper kinase-substrate assignments with equal numbers of random assignments as described above. Both this model and Naïve Bayes+ were applied to perform all-versus-all assignments for kinases with specificity models built from at least 20 substrates against the full high-confidence set of human phosphosites (Ochoa et al., 2020). The numbers of kinases assigned to each phosphosite and the median number of kinases assigned to sites on each substrate protein were analyzed via histograms. All literature-derived assignments in PhosphoSitePlus and all assignments provided by the authors of LinkPhinder at a cutoff of 0.672 were analyzed similarly.

\section{Acknowledgments}

This work was supported by a Wellcome Trust Institutional Strategic Support Award [204904/Z/16/Z]. For the purpose of open access, the author has applied a CC BY public copyright licence to any 
Author Accepted Manuscript version arising from this submission. The author would like to thank Pedro Beltrao for helpful comments on a draft of this manuscript.

\section{References}

Ayati, M., Wiredja, D., Schlatzer, D., Maxwell, S., Li, M., Koyutürk, M., and Chance, M. R. (2019). CoPhosK: A method for comprehensive kinase substrate annotation using cophosphorylation analysis. PLoS Comput Biol, 15(2).

Bachman, J. A., Gyori, B. M., and Sorger, P. K. (2019). Assembling a phosphoproteomic knowledge base using ProtMapper to normalize phosphosite information from databases and text mining. bioRxiv, page 822668 .

Ben Djoudi Ouadda, A., He, Y., Calabrese, V., Ishii, H., Chidiac, R., Gratton, J.-P., Roux, P. P., and Lamarche-Vane, N. (2018). CdGAP/ARHGAP31 is regulated by RSK phosphorylation and binding to 14-3-3 $\beta$ adaptor protein. Oncotarget, 9(14), 11646-11664.

Blom, N., Gammeltoft, S., and Brunak, S. (1999). Sequence and structure-based prediction of eukaryotic protein phosphorylation sites1 1Edited by F. E. Cohen. Journal of Molecular Biology, 294(5), 1351-1362.

Blom, N., Sicheritz-Pontén, T., Gupta, R., Gammeltoft, S., and Brunak, S. (2004). Prediction of post-translational glycosylation and phosphorylation of proteins from the amino acid sequence. PROTEOMICS, 4(6), 1633-1649.

Bradley, D. and Beltrao, P. (2019). Evolution of protein kinase substrate recognition at the active site. PLoS Biol., 17(6), e3000341.

Bradley, D., Viéitez, C., Rajeeve, V., Selkrig, J., Cutillas, P. R., and Beltrao, P. (2021). Sequence and Structure-Based Analysis of Specificity Determinants in Eukaryotic Protein Kinases. Cell Rep, 34(2), 108602.

Brinkworth, R. I., Breinl, R. A., and Kobe, B. (2003). Structural basis and prediction of substrate specificity in protein serine/threonine kinases. PNAS, 100(1), 74-79.

Datta, S. and Mukhopadhyay, S. (2015). A grammar inference approach for predicting kinase specific phosphorylation sites. PLoS One, 10(4), e0122294.

Davis, J. and Goadrich, M. (2006). The relationship between Precision-Recall and ROC curves. In Proceedings of the 23rd International Conference on Machine Learning - ICML '06, pages 233-240, Pittsburgh, Pennsylvania. ACM Press.

Dou, Y., Yao, B., and Zhang, C. (2014). PhosphoSVM: Prediction of phosphorylation sites by integrating various protein sequence attributes with a support vector machine. Amino Acids, 46(6), 1459-1469.

Henikoff, J. G. and Henikoff, S. (1996). Using substitution probabilities to improve positionspecific scoring matrices. Comput. Appl. Biosci., 12(2), 135-143. 
Henikoff, S. and Henikoff, J. G. (1994). Position-based sequence weights. Journal of Molecular Biology, 243(4), 574-578.

Horn, H., Schoof, E. M., Kim, J., Robin, X., Miller, M. L., Diella, F., Palma, A., Cesareni, G., Jensen, L. J., and Linding, R. (2014). KinomeXplorer: An integrated platform for kinome biology studies. Nat Methods, 11(6), 603-604.

Hornbeck, P. V., Zhang, B., Murray, B., Kornhauser, J. M., Latham, V., and Skrzypek, E. (2015). PhosphoSitePlus, 2014: Mutations, PTMs and recalibrations. Nucleic Acids Research, 43(Database-issue), D512-20.

Invergo, B. M. and Beltrao, P. (2018). Reconstructing phosphorylation signalling networks from quantitative phosphoproteomic data. Essays Biochem., 62(4), 525-534.

Invergo, B. M., Petursson, B., Akhtar, N., Bradley, D., Giudice, G., Hijazi, M., Cutillas, P., Petsalaki, E., and Beltrao, P. (2020). Prediction of Signed Protein Kinase Regulatory Circuits. Cell Syst, 10(5), 384-396.e9.

Jung, I., Matsuyama, A., Yoshida, M., and Kim, D. (2010). PostMod: Sequence based prediction of kinase-specific phosphorylation sites with indirect relationship. BMC Bioinformatics, 11(Suppl 1), S10.

Kanshin, E., Kubiniok, P., Thattikota, Y., D’Amours, D., and Thibault, P. (2015). Phosphoproteome dynamics of Saccharomyces cerevisiae under heat shock and cold stress. Mol. Syst. Biol., 11(6), 813.

Kim, J. H., Lee, J., Oh, B., Kimm, K., and Koh, I. (2004). Prediction of phosphorylation sites using SVMs. Bioinformatics, 20(17), 3179-3184.

Krystkowiak, I., Manguy, J., and Davey, N. E. (2018). PSSMSearch: A server for modeling, visualization, proteome-wide discovery and annotation of protein motif specificity determinants. Nucleic Acids Res, 46(W1), W235-W241.

Landry, C. R., Levy, E. D., and Michnick, S. W. (2009). Weak functional constraints on phosphoproteomes. Trends Genet., 25(5), 193-197.

Levy, E. D., Michnick, S. W., and Landry, C. R. (2012). Protein abundance is key to distinguish promiscuous from functional phosphorylation based on evolutionary information. Philosophical Transactions of the Royal Society B: Biological Sciences, 367(1602), 25942606.

Linding, R., Jensen, L. J., Ostheimer, G. J., van Vugt, M. A., Jørgensen, C., Miron, I. M., Diella, F., Colwill, K., Taylor, L., Elder, K., Metalnikov, P., Nguyen, V., Pasculescu, A., Jin, J., Park, J. G., Samson, L. D., Woodgett, J. R., Russell, R. B., Bork, P., Yaffe, M. B., and Pawson, T. (2007). Systematic Discovery of In Vivo Phosphorylation Networks. Cell, 129(7), 1415-1426.

Liu, H. and Grundström, T. (2002). Calcium Regulation of GM-CSF by Calmodulin-Dependent Kinase II Phosphorylation of Ets1. Mol Biol Cell, 13(12), 4497-4507. 
Ma, H., Li, G., and Su, Z. (2020). KSP: An integrated method for predicting catalyzing kinases of phosphorylation sites in proteins. BMC Genomics, 21(1), 537.

Miller, M. L., Jensen, L. J., Diella, F., Jørgensen, C., Tinti, M., Li, L., Hsiung, M., Parker, S. A., Bordeaux, J., Sicheritz-Ponten, T., Olhovsky, M., Pasculescu, A., Alexander, J., Knapp, S., Blom, N., Bork, P., Li, S., Cesareni, G., Pawson, T., Turk, B. E., Yaffe, M. B., Brunak, S., and Linding, R. (2008). Linear Motif Atlas for Phosphorylation-Dependent Signaling. Sci. Signal., 1(35), ra2-ra2.

Nováček, V., McGauran, G., Matallanas, D., Vallejo Blanco, A., Conca, P., Muñoz, E., Costabello, L., Kanakaraj, K., Nawaz, Z., Walsh, B., Mohamed, S. K., Vandenbussche, P.-Y., Ryan, C. J., Kolch, W., and Fey, D. (2020). Accurate prediction of kinase-substrate networks using knowledge graphs. PLoS Comput Biol, 16(12), e1007578.

Obenauer, J. C., Cantley, L. C., and Yaffe, M. B. (2003). Scansite 2.0: Proteome-wide prediction of cell signaling interactions using short sequence motifs. Nucleic Acids Res, 31(13), 3635-3641.

Ochoa, D., Bradley, D., and Beltrao, P. (2018). Evolution, dynamics and dysregulation of kinase signalling. Current Opinion in Structural Biology, 48, 133-140.

Ochoa, D., Jarnuczak, A. F., Viéitez, C., Gehre, M., Soucheray, M., Mateus, A., Kleefeldt, A. A., Hill, A., Garcia-Alonso, L., Stein, F., Krogan, N. J., Savitski, M. M., Swaney, D. L., Vizcaíno, J. A., Noh, K.-M., and Beltrao, P. (2020). The functional landscape of the human phosphoproteome. Nat Biotechnol, 38(3), 365-373.

Rivera, V. M., Miranti, C. K., Misra, R. P., Ginty, D. D., Chen, R. H., Blenis, J., and Greenberg, M. E. (1993). A growth factor-induced kinase phosphorylates the serum response factor at a site that regulates its DNA-binding activity. Mol Cell Biol, 13(10), 6260-6273.

Rodriguez, P., Bhogal, M. S., and Colyer, J. (2003). Stoichiometric Phosphorylation of Cardiac Ryanodine Receptor on Serine 2809 by Calmodulin-dependent Kinase II and Protein Kinase A *. Journal of Biological Chemistry, 278(40), 38593-38600.

Safaei, J., Maňuch, J., Gupta, A., Stacho, L., and Pelech, S. (2011). Prediction of 492 human protein kinase substrate specificities. Proteome Sci, 9(Suppl 1), S6.

Sugiyama, N., Imamura, H., and Ishihama, Y. (2019). Large-scale Discovery of Substrates of the Human Kinome. Scientific Reports, 9(1), 10503.

Szklarczyk, D., Gable, A. L., Lyon, D., Junge, A., Wyder, S., Huerta-Cepas, J., Simonovic, M., Doncheva, N. T., Morris, J. H., Bork, P., Jensen, L. J., and von Mering, C. (2019). STRING v11: Protein-protein association networks with increased coverage, supporting functional discovery in genome-wide experimental datasets. Nucleic Acids Res, 47(D1), D607-D613.

von Mering, C., Jensen, L. J., Snel, B., Hooper, S. D., Krupp, M., Foglierini, M., Jouffre, N., Huynen, M. A., and Bork, P. (2005). STRING: Known and predicted protein-protein associations, integrated and transferred across organisms. Nucleic Acids Res, 33(Database Issue), D433-D437. 
von Stechow, L., Francavilla, C., and Olsen, J. V. (2015). Recent findings and technological advances in phosphoproteomics for cells and tissues. Expert Rev Proteomics, 12(5), 469487.

Wagih, O., Reimand, J., and Bader, G. D. (2015). MIMP: Predicting the impact of mutations on kinase-substrate phosphorylation. Nat. Methods, 12(6), 531-533.

Wagih, O., Sugiyama, N., Ishihama, Y., and Beltrao, P. (2016). Uncovering PhosphorylationBased Specificities through Functional Interaction Networks. Mol. Cell Proteomics, 15(1), 236-245.

Wang, C., Xu, H., Lin, S., Deng, W., Zhou, J., Zhang, Y., Shi, Y., Peng, D., and Xue, Y. (2020). GPS 5.0: An Update on the Prediction of Kinase-specific Phosphorylation Sites in Proteins. Genomics Proteomics Bioinformatics, 18(1), 72-80.

Wang, M., Wang, T., and Li, A. (2017). ksrMKL: A novel method for identification of kinasesubstrate relationships using multiple kernel learning. PeerJ, 5, e4182.

Wright, M. N. and Ziegler, A. (2017). Ranger: A Fast Implementation of Random Forests for High Dimensional Data in C++ and R. Journal of Statistical Software, 77(1), 1-17.

Xue, Y., Liu, Z., Cao, J., Ma, Q., Gao, X., Wang, Q., Jin, C., Zhou, Y., Wen, L., and Ren, J. (2011). GPS 2.1: Enhanced prediction of kinase-specific phosphorylation sites with an algorithm of motif length selection. Protein Eng Des Sel, 24(3), 255-260.

Yaffe, M. B., Leparc, G. G., Lai, J., Obata, T., Volinia, S., and Cantley, L. C. (2001). A motifbased profile scanning approach for genome-wide prediction of signaling pathways. Nature Biotechnology, 19(4), 348-353.

Yang, P., Humphrey, S. J., James, D. E., Yang, Y. H., and Jothi, R. (2016). Positive-unlabeled ensemble learning for kinase substrate prediction from dynamic phosphoproteomics data. Bioinformatics, 32(2), 252-259.

Yang, S., Ji, M., Zhang, L., Chen, Y., Wennmann, D. O., Kremerskothen, J., and Dong, J. (2014). Phosphorylation of KIBRA by the extracellular signal-regulated kinase (ERK) ribosomal S6 kinase (RSK) cascade modulates cell proliferation and migration. Cell Signal, 26(2), 343-351.

Zhang, M.-L., Peña, J. M., and Robles, V. (2009). Feature selection for multi-label naive Bayes classification. Information Sciences, 179(19), 3218-3229.

Zhou, F.-F., Xue, Y., Chen, G.-L., and Yao, X. (2004). GPS: A novel group-based phosphorylation predicting and scoring method. Biochemical and Biophysical Research Communications, 325(4), 1443-1448.

Zou, L., Wang, M., Shen, Y., Liao, J., Li, A., and Wang, M. (2013). PKIS: Computational identification of protein kinases for experimentally discovered protein phosphorylation sites. BMC Bioinformatics, 14, 247. 